\title{
Biométrie des enfants de 0 à 5 ans issus d'une famille défavorisée à Abidjan
}

\author{
Kouadio Kouakou Jérôme (Docteur, Maître-Assistant) \\ Unité Pédagogique et de Recherches en Paléoanthropologie, \\ Institut des Sciences Anthropologiques de Développement (ISAD), \\ Université Félix Houphouët-Boigny d'Abidjan Cocody (UFHB). \\ Abidjan, Côte d'Ivoire
}

Doi:10.19044/esj.2019.v15n2p139 ～URL:http://dx.doi.org/10.19044/esj.2019.v15n2p139

\section{Résumé}

Le développement physique de l'enfant semble dépendre, entre autres, des conditions de vie qui lui sont offertes. Ces circonstances d'existence pourraient ainsi varier d'un pays à l'autre. L'influence de celles proposées à ceux vivant Abidjan sur leurs acquisitions physiques est loin d'être suffisamment examinée. Le présent travail compare le développement physique des enfants de Milieu Familial Défavorisé (MFD) à celui de leurs pairs bénéficiant d'un Milieu Familial Favorisé (MFF). Il porte sur 278 enfants âgés de 6 à 59 mois dont 136 issus du MFD et 142 sélectionnés dans un MFF. L'examen du développement physique des deux groupes d'enfants s'est fait en considérant le poids, la stature et le périmètre crânien moyens. La balance électronique et le ruban mètre sont utilisés pour la mesure des caractères corporels évoqués. Les résultats montrent, à l'issue des comparaisons des caractères corporels, que les nourrissons et les enfants qui vivent dans un MFD présentent un développement physique qui ne diffère significativement pas de celui de leurs pairs issus de MFF. Ces résultats sont loin de confirmer l'hypothèse selon laquelle le statut socio-économique du milieu familial crée des différences au plan du développement physique des enfants.

Mots-clés: Côte d'Ivoire, Développement physique, Enfant, Famille, Nourrisson 


\title{
Biometrics of children aged 0 to 5 from a disadvantaged family in Abidjan
}

\author{
Kouadio Kouakou Jérôme (Docteur, Mâ̂tre-Assistant) \\ Unité Pédagogique et de Recherches en Paléoanthropologie, \\ Institut des Sciences Anthropologiques de Développement (ISAD), \\ Université Félix Houphouët-Boigny d'Abidjan Cocody (UFHB). \\ Abidjan, Côte d'Ivoire
}

\begin{abstract}
The body growth of the child seems to depend, among other things, on the living conditions that are offered to him. These circumstances of existence could thus vary from one country to another. The influence of those proposed to those living in Abidjan on their bodily acquisitions is far from being sufficiently examined. This paper compares the body growth of children in disadvantaged family environments (MFD) with their peers with a Favored Family Environment (FLM).It covers 278 children aged 6 to 59 months, including 136 from the MFD and 142 selected from a FLM. Examination of the body growth of the two groups of children was based on average weight, stature, and head circumference. The electronic scale and the tape measure are used to measure evoked body characteristics. The results show, after body character comparisons, that infants and children who live in a MFD exhibit a body growth that does not differ significantly from that of their FLM peers. These results are far from confirming the hypothesis that the socio-economic status of the home environment creates differences in children's physical development.
\end{abstract}

Keywords: Child, Côte d'Ivoire, Family, Infant, Body Growth

\section{Introduction}

L'enfant, d'un âge à l'autre, subit des transformations corporelles. L'ensemble de celles-ci est désigné sous le vocable de développement physique. Selon Amor et al. (2001), celui-ci renvoie à un processus de modifications des caractères physiques de l'enfant. Il est considéré, par Froment et Koppert (2000), comme l'acquisition progressive de fonctions biophysiques sous l'effet du milieu. Le développement physique serait donc le produit de l'interaction entre les caractères biophysiologiques de l'enfant et son milieu de vie. 
L'influence du milieu de vie de l'enfant sur le développement physique de celui-ci serait d'autant probable que, selon Tano (2000), les enfants ne bénéficient pas toujours pas du même environnement physique et socioculturel. Par exemple, en Côte d'Ivoire, le père est celui qui offre, le plus souvent, à l'enfant, une maison, et apporte à celle-ci les ressources économiques et matérielles pour l'épanouissement de cet enfant, notamment un dortoir, de l'eau, des aliments. La mère, quant à elle, assure à l'enfant, dans nombre de cas, sa diète journalière, ses soins sanitaires, ses besoins affectifs et de sécurité (Landry, 2014).

De ce point de vue, en fonction des possibilités socio-économiques des parents, l'enfant peut bénéficier d'un cadre de vie et des soins singuliers. Si les parents possèdent de bonnes et suffisantes ressources, l'enfant pourrait disposer d'un dortoir aéré et sain, d'un lieu de bain hygiénique, d'une eau potable, d'une alimentation suffisante, équilibrée, des soins sanitaires adaptés et ajustés à son âge et à son sexe. Il vit, ce faisant, dans un milieu familial que l'on pourrait appeler favorisé.

Les stimulations positives que procure un tel milieu familial ne sont pas loin de favoriser chez l'enfant un bon fonctionnement biophysiologique, une meilleure expression de son potentiel interne et, conséquemment, un développement physique de qualité. En d'autres termes, le milieu familial favorisé créerait des conditions alimentaires et sanitaires nécessaires à un développement physique harmonieux.

En revanche, si les parents ont moins de moyens socioéconomiques, l'enfant interagirait avec un milieu qui ne pourrait lui procurer, d'une part, un cadre pour un sommeil réparateur, et, d'autre part, lui offrir une alimentation équilibrée, saine et une eau de boisson préservée de contamination bactérienne. Les ressources pourvues par ce milieu familial défavorisé seraient d'autant moins bénéfiques que, selon Hardoy et al. (2001), les déchets solides et liquides, produits dans un tel milieu, sont déversés à proximité des habitations. Le milieu familial défavorisé se caractériserait donc par une atmosphère de vie emprunte d'insalubrité, de pollution et de toxicité régulière.

Ces mauvaises stimulations pourraient exposer l'enfant à des maladies à transmission vectorielle (diarrhée, paludisme, dysenterie, anémie), c'est-àdire des maladaptations biophysiologiques. De tels dysfonctionnements internes sont susceptibles d'être préjudiciables au développement physique de l'enfant. La variabilité de ce développement aurait pour source la non similarité des conditions de vie offertes aux enfants par les familles.

$\mathrm{La}$ relation entre les variables socio-environnementales et le développement physique, a fait objet de divers travaux. Par exemple, Hioui et al. (2009), Hermanussen et Bogin (2014) ont examiné l'influence des conditions socio-économiques des familles sur le développement physique de l'enfant. Dans cette optique, les aliments que l'enfant de moins de cinq ans 
reçoit ont été considérés comme exerçant des effets variables sur les acquisitions corporelles en fonction de la quantité et la qualité de ces ressources alimentaires (Shinsugi et al.2015). Ainsi, Sana et al. (2012) ont montré que le milieu physique, socio-culturel de l'enfant occasionne chez ce dernier un développement physique spécifique. La trajectoire corporelle de l'enfant pourrait donc être façonnée, entre autres, par les conditions socioéconomique et physique du milieu familial dans lequel ce dernier vit.

Or, il semble que, dans les recherches précédemment évoquées, l'accent n'ait pas suffisamment été mis sur les stimulations socioéconomiques du milieu familial proposé aux enfants vivant à Abidjan en rapport avec leur développement physique. L'objectif de cette étude est d'examiner le développement physique des enfants abidjanais issus de milieux familiaux de statuts socio-économiques différents.

\section{Méthodologie}

\section{1. Matériel}

Le présent travail s'interroge sur le développement physique des enfants issus de milieu familial défavorisé. En effet, les enfants qui naissent dans un milieu parental moins avantageux semblent constituer une proportion importante par rapport aux naissances d'une année. La plupart de ces enfants de conditions défavorables est originaire des pays en développement (Ayissi et al., 2002; Keino et al., 2014). Des observations faites, par exemple en Côte d'Ivoire, montrent que deux tiers des enfants naissent dans des milieux modestes (Talnan et al., 2008).

Les grandes agglomérations ivoiriennes se révèlent être celles dans lesquelles ces enfants sont en nombre important. L'on fait état, dans ce sens, d'une fréquence non négligeable de ces enfants dans la ville d'Abidjan, où plus de 50\% d'entre eux résident dans les communes se caractérisant par une population à faible revenu (Talnan et al., 2008). L'on note également que 39\% de ces enfants de moins de cinq ans présentent, généralement, un retard de croissance corporelle (Institut National de la Statistique (INS, 2012).

Dans ce cas et conformément aux indications de Schwartz (1963), la taille de l'échantillon à observer pourrait être estimée à partir de ce taux, en considérant la formule suivante. Taille de l'échantillon $(\mathrm{N}): \mathrm{N}=\mathrm{t}^{2} \times \mathrm{p}(1-\mathrm{p})$ / $\mathrm{m}^{2} . \mathrm{N}=$ taille d'échantillon requise, $\mathrm{t}=$ niveau de confiance à $95 \%$ (valeur type de 1,96), $\mathrm{p}=$ prévalence estimative de la malnutrition dans la zone d'étude $(12 \%$ ou 0,12$), \mathrm{m}=$ marge d'erreur à $5 \%$ (valeur type de 0,05$) . \mathrm{N}=1.96^{2} \times 0,39$ $(1-0,39) / 0,05^{2}=366$ sujets.

L'indisponibilité d'une base de données portant sur ces enfants et le refus de plusieurs parents ne permettent ni une sélection probabiliste des sujets ni de retenir la taille de l'échantillon précédemment indiquée. Par conséquent, un échantillon, par convenance, d'enfants est constitué, après avoir obtenu le 
consentement éclairé des parents. Cet échantillonnage (au jugé) s'est effectué dans deux communes d'Abidjan qui abritent des enfants vivant dans des familles de statut socio-économique défavorisé ou favorisé, en l'occurrence celles de Koumassi et de Marcory.

Située dans la zone sud d'Abidjan, la commune de Koumassi est une des dix qui structurent la ville d'Abidjan. Elle s'étend sur une superficie de 874 hectares. Koumassi est entourée au nord par la commune de Marcory, au sud par celle de Port-Bouët, au sud-est et au nord-est par la lagune Ebrié. Elle est, de ce fait, une presqu'île qui abrite 433139 habitants (INS, 2014). L'habitat est implanté sur environ $45 \%$ de la surface communale dont $26 \%$ est constituée d'habitats précaires. Ceux-ci sont situés dans les quartiers situés au sud de la commune Grand campement, Akomiabla, Yapokro, HouphouëtBoigny 1 et 2, qui comprennent au total 10546 baraques et 160 cases traditionnelles (INS, 1998). Outre sa structuration socio-économique défavorisée, l'on compte dans cette commune un effectif de 7131 enfants âgés de 0 à 59 mois.

Ces enfants sont composés de filles et de garçons dont les parents (père, mère) exercent presque tous des activités d'ouvriers, de commerçants, de conducteurs d'automobiles de transport. En outre, l'on note que ces enfants vivent dans des familles de grande taille (7 ou 8 enfants) et sont soumis à une alimentation constituée essentiellement de féculents et de céréales à moindre coût, moins consommés dans des quartiers favorisés. Ainsi, la situation de vie de ces enfants est loin d'être identique à celle de leurs homologues nés dans des milieux avantageusement structurés.

Tenant compte de la proximité géographique, les enfants de 0 à 5 ans, nés à Marcory, une commune abidjanaise abritant des familles relativement favorisées, constituent le second sous-échantillon examiné dans le présent travail. Marcory est une autre commune d'Abidjan. Située sur le périmètre de l'Ile de Petit-Bassam, elle est limitée au sud par la commune de Koumassi, au nord par la lagune Ebrié, à l'ouest par celle de Treichville. Comparativement à Koumassi, Marcory est occupée par les quartiers résidentiels qui logent les habitants dont le niveau socio-économique est élevé. L'infrastructure routière y est bien développée et cette zone héberge une population estimée à 249858 habitants dont 2428 enfants de 0 à 5 ans (INS, 2014; Müller et al., 2010).

Ces derniers sont nés de parents qui sont, pour la plupart, des cadres d'entreprises, des enseignants du supérieur, des cadres de l'administration publique, des hauts entrepreneurs. Ainsi, ils bénéficient de famille de petite taille ( 2 ou 3 enfants) et d'une alimentation variée (céréales et féculents de qualité, fruits, légumes, légumineuses). Le quartier de Marcory dispose des équipements de stockage de l'eau de boisson, d'évacuation des eaux usées et de stockage des ordures ménagères salubres et régulièrement renouvelés que ceux de la bourgade défavorisée de Koumassi. 
Considérant ces caractéristiques sociodémographiques et économiques comme critères d'inclusion ou de non inclusion, deux groupes d'enfants sont retenus. Le premier groupe compte 136 enfants de famille défavorisée dont 70 garçons et 66 filles. Le second groupe (favorisé) comprend 70 garçons et 72 filles. En somme, 278 enfants âgés de 6 à 59 mois dont 136 de conditions de vie moins bonnes et 142 bénéficiant de possibilités de vie relativement appropriées constituent l'échantillon sur lequel porte l'étude.

\section{2. Méthodes}

L'étude est une observation transversale analytique. Elle s'intéresse au développement physique des enfants en lien avec le type de milieu familial dans lequel ces derniers vivent. Ainsi, des enfants, issus des communes de Koumassi et Marcory, ont été observés selon différentes étapes. D'abord, l'on a administré à 395 femmes sélectionnées au jugé vivant dans ces deux communes et possédant un nouveau-né, un nourrisson ou un enfant postnourrisson un questionnaire composé de 45 questions portant aussi bien sur les caractéristiques sociodémographiques et économiques de la famille. Cette étape s'est réalisée avec le consentement des mères.

Le dépouillement des réponses données aux divers items par ces mères a permis de sélectionner 363 enfants, âgés de 6 à 59 mois, dont les mères sont âgées de 26 à 35 ans. Cent quatre-vingt-six (186) de ces enfants sont de milieu familial défavorisé (Koumassi) et cent soixante-dix-sept (177) de milieu familial favorisé (Marcory).

Ensuite, ces groupes ont été, après l'autorisation de leurs mères, soumis à des mesures corporelles, en l'occurrence le poids, la stature et le périmètre céphalique. L'absence de quelques mères ainsi que la difficulté à disposer des enfants n'ont pas favorisé la participation de la totalité des enfants préalablement sélectionnés à la seconde étape de l'observation des enfants. Cette étape a donc été réalisée auprès de 278 enfants d'âge compris entre 6 et 59 mois dont 136 sont issus de milieu socio-sanitaire défavorisé et 142 de milieu socio-sanitaire favorisé.

Ainsi, la stature des deux groupes d'enfants, conformément aux indications de World Health Organization (2006), est explorée au moyen d'une toise portable de fabrication locale. Le périmètre crânien de ces nourrissons et post-nourrissons, quant à lui, est obtenu par l'utilisation d'un ruban. Ces caractères corporels des enfants sont mesurés avec une précision de $0.1 \mathrm{~cm}$. Le poids a été mesuré au kilogramme près par le truchement d'une balance analogique, avec une précision de $0.5 \mathrm{~kg}$. Cette triple opération, qui a duré 3 mois, a permis d'obtenir des données sur le développement physique des deux groupes de nourrissons et de post-nourrissons considérés. La saisie et le nettoyage des données sont faits en se servant des logiciels Nutrisurvey Anthro v3.2.2 (2005. exe); Excel et SPSS (version 23.0) pour Windows. La distribution de ces données corporelles structurées est examinée 
statistiquement en appliquant le test de Kolmogorov Smirnov. Les résultats de ce contrôle montrent que les données pondérales, staturales et céphaliques ne suivent pas, dans la plupart des cas, une distribution normale ( $p$-value $=0,200$; $\mathrm{p}$-value $=0,063 \mathrm{p}$-value $=0,037$ ).

Enfin, les données relatives aux dimensions corporelles présentées par les deux groupes d'enfants ont été présentées sous forme de scores moyens. L'analyse statistique, enfin, de ces scores s'est effectuée au moyen du test non paramétrique, le test $U$ de Mann-Withney, pour tenir compte de la non normalité de la distribution des données corporelles.

L'emploi de ce test se révèle nécessaire pour la comparaison des données quantitatives mesurant le développement physique des enfants selon les milieux familiaux dans lesquels ces nourrissons et enfants vivent. Dans ce cadre, le logiciel Statistical Package of Social Science (SPSS) a été 1'outil choisi pour le traitement de ces données. Le seuil, à partir duquel la différence entre les sujets est significative, considérant la nature des données (non biologiques), est fixé à 0,05 , un référentiel conventionnel.

\section{Résultats}

Le développement physique, notamment le poids, la taille, et le périmètre crânien des enfants âgés de 6 à 59 mois issus de milieu sociosanitaire défavorisé ont été comparés à ceux de leurs pairs de milieu sociosanitaire favorisé. Ainsi, les nourrissons de 6 à 10 mois qui vivent dans un milieu familial défavorisé présentent un poids, une stature et un périmètre crânien moyens qui ne diffèrent statistiquement pas de ceux de leurs homologues bénéficiant d'un milieu familial favorisé $(7,51 \mathrm{~kg}$ contre 8,23 kg; $69,70 \mathrm{~cm}$ contre $71,69 \mathrm{~cm} ; 43,66 \mathrm{~cm}$ contre $44,40 \mathrm{~cm})$.

Tableau I: Comparaison du poids $(\mathrm{kg})$, de la stature $(\mathrm{cm})$ et du périmètre crânien $(\mathrm{cm})$ moyens chez les nourrissons de 6 à 10 mois de Milieu Familial Défavorisé (MFD) à ceux de leurs pairs de Milieu Familial Favorisé (MFF)

\begin{tabular}{|c|c|c|c|c|c|c|c|c|}
\hline $\begin{array}{l}\text { Milieu } \\
\text { Familial }\end{array}$ & Sexe & $\mathrm{N}$ & Poids & $\mathrm{p}$-value & Stature & $\mathrm{p}$-value & PC & $\mathrm{p}$-value \\
\hline MFD & $\mathrm{G}$ & 4 & $\begin{array}{c}7.85 \\
\pm 0,14 \\
\end{array}$ & \multirow[t]{2}{*}{$0,489 \mathrm{~ns}$} & $\begin{array}{c}70.08 \\
\pm 3,42 \\
\end{array}$ & \multirow[t]{2}{*}{$0,571 \mathrm{~ns}$} & $\begin{array}{l}43.58 \\
\pm 0,60\end{array}$ & \multirow[t]{2}{*}{$1,000 \mathrm{~ns}$} \\
\hline & $\mathrm{F}$ & 6 & $\begin{array}{c}7.17 \\
\pm 0.23 \\
\end{array}$ & & $\begin{array}{c}69.32 \\
\pm .0 .07 \\
\end{array}$ & & $\begin{array}{l}43.75 \\
\pm 0,65 \\
\end{array}$ & \\
\hline \multirow[t]{2}{*}{ MFF } & G & 6 & $\begin{array}{c}8.07 \\
\pm 0,04\end{array}$ & \multirow[t]{2}{*}{$0,548 \mathrm{~ns}$} & $\begin{array}{r}71.37 \\
\pm 0,52\end{array}$ & \multirow[t]{2}{*}{$0,905 \mathrm{~ns}$} & $\begin{array}{l}43.90 \\
\pm 0,69\end{array}$ & \multirow[t]{2}{*}{$0,548 \mathrm{~ns}$} \\
\hline & $\mathrm{F}$ & 4 & $\begin{array}{c}8.40 \\
\pm 0,03 \\
\end{array}$ & & $72 \pm 2,68$ & & $\begin{array}{l}44.90 \\
\pm 0,21 \\
\end{array}$ & \\
\hline $\begin{array}{c}\text { Mann- } \\
\text { Withney }\end{array}$ & & & $0.105 \mathrm{~ns}$ & & $0.05 \mathrm{~ns}$ & & $0.460 \mathrm{~ns}$ & \\
\hline
\end{tabular}

N:Effectif des sujets; G: Garçon; F: Fille; PC: Périmètre crânien; S: Différence statistiquement Significative; NS: Différence Non statistiquement Significative 
Des résultats analogues sont observés chez les deux groupes de nourrissons de 11 à 15 mois, $(8,90 \mathrm{~kg}$ contre $8,86 \mathrm{~kg} ; 73,07 \mathrm{~cm}$ contre 73,79 $\mathrm{cm} ; 44,86 \mathrm{~cm}$ contre 45,94 cm) (confère le tableau II).

Tableau II: Comparaison du poids $(\mathrm{kg})$, de la stature $(\mathrm{cm})$ et du périmètre crânien $(\mathrm{cm})$ moyens chez les nourrissons de 11 à 15 mois de MFD à ceux de leurs pairs de MFF

\begin{tabular}{|c|c|c|c|c|c|c|c|c|}
\hline Milieu Familial & Sexe & $\mathrm{N}$ & Poids & p-value & Stature & $\mathrm{p}$-value & $\mathrm{PC}$ & $\mathrm{p}$-value \\
\hline \multirow[t]{2}{*}{ MFD } & $\mathrm{G}$ & 4 & $\begin{array}{c}9.50 \\
\pm 0,07\end{array}$ & \multirow{2}{*}{$0,181 \mathrm{~ns}$} & $\begin{array}{l}72.70 \\
\pm 0,21\end{array}$ & \multirow[t]{2}{*}{$0,573 \mathrm{~ns}$} & $\begin{array}{c}44.83 \\
\pm 0,02\end{array}$ & \multirow[t]{2}{*}{$0,491 \mathrm{~ns}$} \\
\hline & $\mathrm{F}$ & 6 & $\begin{array}{c}8.3 \\
\pm 1,13 \\
\end{array}$ & & $\begin{array}{r}73.43 \\
\pm 1,02 \\
\end{array}$ & & $\begin{array}{l}44.89 \\
\pm 1,75 \\
\end{array}$ & \\
\hline \multirow[t]{2}{*}{ MFF } & G & 4 & $\begin{array}{c}9.18 \\
\pm 0,45\end{array}$ & \multirow[t]{2}{*}{$0,151 \mathrm{~ns}$} & $\begin{array}{c}73.7 \\
\pm 1,69\end{array}$ & \multirow[t]{2}{*}{$0,310 \mathrm{~ns}$} & $\begin{array}{c}45.77 \\
\pm 0,84\end{array}$ & \multirow[t]{2}{*}{$1,000 \mathrm{~ns}$} \\
\hline & $\mathrm{F}$ & 5 & $\begin{array}{c}8.55 \\
\pm 0,21\end{array}$ & & $\begin{array}{c}73.88 \\
\pm 0,53\end{array}$ & & $\begin{array}{l}46.12 \\
\pm 0,26\end{array}$ & \\
\hline Mann-Withney & & & $0.243 \mathrm{~ns}$ & & $0.447 \mathrm{~ns}$ & & $0.606 \mathrm{~ns}$ & \\
\hline
\end{tabular}

N:Effectif des sujets; G: Garçon; F: Fille; PC: Périmètre crânien; S: Différence statistiquement Significative; NS: Différence Non statistiquement Significative.

Les résultats ne changent pas, lorsque l'on examine le poids, la stature et le périmètre crânien moyens des nourrissons de 16 à 24 mois. Ceux de MFD ne diffèrent pas de leurs homologues de MFF, du point de vue de la croissance corporelle $(9,33 \mathrm{~kg}$ contre $9,87 \mathrm{~kg} ; 78,19 \mathrm{~cm}$ contre 79,$86 ; 45,89 \mathrm{~cm}$ contre $46,24 \mathrm{~cm}$ ) (confère le tableau III).

Tableau III: Comparaison du poids $(\mathrm{kg})$, de la stature $(\mathrm{cm})$ et du périmètre crânien $(\mathrm{cm})$ moyens chez les nourrissons de 16 à 20 de MFD à ceux de leurs pairs de MFF

\begin{tabular}{|c|c|c|c|c|c|c|c|c|}
\hline Milieu Familial & Sexe & $\mathrm{N}$ & Poids & p-value & Stature & p-value & $\mathrm{PC}$ & p-value \\
\hline MFD & $G$ & 3 & $\begin{array}{c}9.60 \\
\pm 0,28 \\
\end{array}$ & \multirow[t]{2}{*}{$0,582 \mathrm{~ns}$} & $\begin{array}{r}79.70 \\
\pm 0,98 \\
\end{array}$ & \multirow[t]{2}{*}{$0,073 \mathrm{~ns}$} & $\begin{array}{l}45.87 \\
\pm 0,77 \\
\end{array}$ & \multirow[t]{2}{*}{$0,582 \mathrm{~ns}$} \\
\hline & $\mathrm{F}$ & 10 & $\begin{array}{c}9.06 \\
\pm 0,44\end{array}$ & & $\begin{array}{l}76.68 \\
\pm 0,31\end{array}$ & & $\begin{array}{l}45.91 \\
\pm 0,70\end{array}$ & \\
\hline \multirow[t]{2}{*}{ MFF } & G & 4 & $\begin{array}{c}10.27 \pm \\
1,03 \\
\end{array}$ & \multirow[t]{2}{*}{$0,230 \mathrm{~ns}$} & $\begin{array}{c}80.92 \pm \\
2,94 \\
\end{array}$ & \multirow[t]{2}{*}{$0,230 \mathrm{~ns}$} & $\begin{array}{r}46.20 \\
\pm 0,38 \\
\end{array}$ & \multirow[t]{2}{*}{$0,412 \mathrm{~ns}$} \\
\hline & $\mathrm{F}$ & 6 & $\begin{array}{c}9.47 \\
\pm 0,02 \\
\end{array}$ & & $\begin{array}{r}78.83 \\
\pm 0,02 \\
\end{array}$ & & $\begin{array}{l}46.28 \\
\pm 0,09 \\
\end{array}$ & \\
\hline Mann-Withney & & & $0.284 \mathrm{~ns}$ & & $0.077 \mathrm{~ns}$ & & $0.257 \mathrm{~ns}$ & \\
\hline
\end{tabular}

N: effectif des sujets; G: Garçon; F: Fille; PC: Périmètre crânien; S: Différence statistiquement Significative; NS: Différence Non statistiquement Significative

Le rythme du développement corporel des nourrissons ne varie pas d'un âge à l'autre. La comparaison des caractères corporels des nourrissons de 16 à 20 mois de MFD à ceux de leurs pairs de MFF montrent une équivalence entre les deux groupes de nourrissons $(10,005 \mathrm{~kg}$ contre $10,62 \mathrm{~kg} ; 80,75 \mathrm{~cm}$ contre $81,10 \mathrm{~cm} ; 46,80 \mathrm{~cm}$ contre 46,43 cm) (confère le tableau IV). 
Tableau IV: Comparaison du poids $(\mathrm{kg})$, de la stature $(\mathrm{cm})$ et du périmètre crânien $(\mathrm{cm})$ moyens chez les nourrissons de 21 à 24 mois de MFD à ceux de leurs pairs de MFF

\begin{tabular}{|c|c|c|c|c|c|c|c|c|}
\hline Milieu Familial & Sexe & $\mathrm{N}$ & Poids & $\mathrm{p}$-value & Stature & $\mathrm{p}$-value & $\mathrm{PC}$ & $\mathrm{p}$-value \\
\hline MFD & $\bar{G}$ & 3 & $\begin{array}{r}10.17 \\
\pm 0.02\end{array}$ & \multirow{2}{*}{$\begin{array}{c}0,250 \\
\mathrm{~ns}\end{array}$} & $\begin{array}{c}81.87 \\
\pm 0.09\end{array}$ & \multirow{2}{*}{$0,393 \mathrm{~ns}$} & $\begin{array}{c}46.73 \\
\pm 0.02\end{array}$ & \multirow{2}{*}{$0,393 \mathrm{~ns}$} \\
\hline & $\mathrm{F}$ & 4 & $\begin{array}{c}9.84 \\
\pm 0,23\end{array}$ & & $\begin{array}{c}79.62 \pm \\
0,12\end{array}$ & & $\begin{array}{c}45.82 \\
\pm 0,30\end{array}$ & \\
\hline \multirow[t]{2}{*}{ MFF } & $G$ & 6 & $\begin{array}{c}11.49 \\
\pm 0,44\end{array}$ & \multirow{2}{*}{$0,017 \mathrm{~s}$} & $\begin{array}{r}83.20 \\
\pm 1,17\end{array}$ & \multirow{2}{*}{$0,017 \mathrm{~s}$} & $\begin{array}{c}46.87 \\
\pm 1,32\end{array}$ & \multirow{2}{*}{$0,183 \mathrm{~ns}$} \\
\hline & $\mathrm{F}$ & 3 & $\begin{array}{c}9.75 \\
\pm 0,35\end{array}$ & & $\begin{array}{c}79 \\
\pm 0,88\end{array}$ & & $\begin{array}{c}46 \\
\pm 0,07\end{array}$ & \\
\hline Mann-Withney & & & $0.091 \mathrm{~ns}$ & & $\begin{array}{c}0.536 \\
n s\end{array}$ & & $0.055 \mathrm{~ns}$ & \\
\hline
\end{tabular}

N: effectif des sujets; G: Garçon; F: Fille; PC: Périmètre crânien; S: Différence statistiquement Significative; NS: Différence Non statistiquement Significative

À l'âge d'enfant, les sujets issus des deux milieux familiaux sont superposables. Par exemple, les enfants de 25 à 30 ans issus du MFD se caractérisent par un poids, une stature et un périmètre crânien qui ne s'écartent pas statistiquement de ceux de leurs pairs vivant un MFF $(10,91 \mathrm{~kg}$ contre $11,96 \mathrm{~kg} ; 84,91 \mathrm{~cm}$ contre $87,79 \mathrm{~cm} ; 46,81 \mathrm{~cm}$ contre 46,90 cm) (confère le tableau V).

Tableau V: Comparaison du poids $(\mathrm{kg})$, de la stature $(\mathrm{cm})$ et du périmètre crânien $(\mathrm{cm})$

moyens chez les enfants âgés de 25 à 30 mois de MFD à ceux de leurs pairs de MFF

\begin{tabular}{|c|c|c|c|c|c|c|c|c|}
\hline Milieu Familial & Sexe & $\mathrm{N}$ & Poids & $\mathrm{p}$-value & Stature & $\mathrm{p}$-value & PC & p-value \\
\hline MFD & $G$ & 13 & $\begin{array}{c}10.51 \\
\pm 0,22\end{array}$ & \multirow{2}{*}{$\begin{array}{c}0,091 \\
\text { ns }\end{array}$} & $\begin{array}{r}82.99 \\
\pm 3,69\end{array}$ & \multirow[t]{2}{*}{$0,044 \mathrm{~s}$} & $\begin{array}{l}46.52 \\
\pm 0,35\end{array}$ & \multirow[t]{2}{*}{$0,840 \mathrm{~ns}$} \\
\hline & $\mathrm{F}$ & 13 & $\begin{array}{c}11.31 \\
\pm 0,19 \\
\end{array}$ & & $\begin{array}{r}86.84 \\
\pm 0,18 \\
\end{array}$ & & $\begin{array}{l}47.10 \\
\pm 0,19 \\
\end{array}$ & \\
\hline \multirow[t]{2}{*}{ MFF } & $G$ & 16 & $\begin{array}{r}12.19 \\
\pm 0.24\end{array}$ & \multirow[b]{2}{*}{$\begin{array}{c}0,232 \\
\mathrm{~ns}\end{array}$} & $\begin{array}{r}88.49 \\
\pm 0.53\end{array}$ & \multirow{2}{*}{$0,188 \mathrm{~ns}$} & $\begin{array}{l}46.80 \\
\pm 0.09\end{array}$ & \multirow{2}{*}{$0,830 \mathrm{~ns}$} \\
\hline & $\mathrm{F}$ & 15 & $\begin{array}{c}11.74 \\
\pm 0,44\end{array}$ & & $\begin{array}{l}87.09 \\
\pm 0,72\end{array}$ & & $47 \pm 0,14$ & \\
\hline Mann-Withney & & & $0.031 \mathrm{~s}$ & & $0.052 \mathrm{~ns}$ & & $0.495 \mathrm{~ns}$ & \\
\hline
\end{tabular}

N: Effectif des sujets; G: Garçon; F: Fille; PC: Périmètre crânien; S: Différence statistiquement Significative; NS: Différence Non statistiquement Significative

La croissance de ces deux groupes d'enfants ne varie à l'âge de 31 à 36 mois. L'on constate que les caractères biométriques de ces enfants se situent à un niveau de développement similaire $(12,33 \mathrm{~kg}$ contre $13,25 \mathrm{~kg}$; $90,13 \mathrm{~cm}$ contre $92,38 \mathrm{~cm} ; 48,03 \mathrm{~cm}$ contre 49,05 cm) (confère le tableau VI). 
Tableau VI: Comparaison du poids $(\mathrm{kg})$, de la stature $(\mathrm{cm})$ et du périmètre crânien $(\mathrm{cm})$ moyens chez les enfants âgés 31 à 36 mois de MFD à ceux de leurs pairs de MFF

\begin{tabular}{|c|c|c|c|c|c|c|c|c|}
\hline Milieu Familial & Sexe & $\mathrm{N}$ & Poids & $\begin{array}{c}\mathrm{p}- \\
\text { value }\end{array}$ & Stature & $\mathrm{p}$-value & PC & $\mathrm{p}$-value \\
\hline MFD & G & 11 & $\begin{array}{c}12.32 \\
\pm 0,68\end{array}$ & \multirow{2}{*}{$\begin{array}{c}0,404 \\
\text { ns }\end{array}$} & $\begin{array}{l}88.82 \\
\pm 0,16\end{array}$ & \multirow{2}{*}{$\begin{array}{c}0,808 \\
\text { ns }\end{array}$} & $\begin{array}{l}48.46 \\
\pm 0,06\end{array}$ & \multirow[t]{2}{*}{$0,036 \mathrm{~s}$} \\
\hline & $\mathrm{F}$ & 6 & $\begin{array}{c}12.34 \\
\pm 0,47\end{array}$ & & $\begin{array}{l}91.44 \\
\pm 0,36\end{array}$ & & $\begin{array}{l}47.59 \\
\pm 0,37\end{array}$ & \\
\hline \multirow[t]{2}{*}{ MFF } & G & 8 & $\begin{array}{c}13.30 \\
\pm 0,37\end{array}$ & \multirow{2}{*}{$\begin{array}{c}0,346 \\
\text { ns }\end{array}$} & $\begin{array}{c}92.43 \\
\pm 0,55\end{array}$ & \multirow{2}{*}{$\begin{array}{c}0,582 \\
\mathrm{~ns}\end{array}$} & $\begin{array}{c}49.14 \\
\pm 0,04\end{array}$ & \multirow[t]{2}{*}{$0,872 \mathrm{~ns}$} \\
\hline & $\mathrm{F}$ & 12 & $\begin{array}{c}13.2 \pm \\
0,35\end{array}$ & & $\begin{array}{c}92.33 \\
\pm 2,96\end{array}$ & & $\begin{array}{l}48.97 \\
\pm 0.42\end{array}$ & \\
\hline Mann-Withney & & & $0.048 \mathrm{~ns}$ & & $0.149 \mathrm{~ns}$ & & $0.016 \mathrm{~ns}$ & \\
\hline
\end{tabular}

N: Effectif des sujets; G: Garçon; F: Fille; PC: Périmètre crânien; S: Différence statistiquement Significative; NS: Différence Non statistiquement Significative

Aux alentours de 4 mois, la croissance physique des enfants dont les parents sont défavorisés ressemblent à leurs pairs de parents favorisés. La comparaison des poids, statures et périmètres crâniens des deux groupes enfants illustrent cette trajectoire corporelle indifférenciée $(12,92 \mathrm{~kg}$ contre $14,88 \mathrm{~kg} ; 96,93 \mathrm{~cm}$ contre $99,41 \mathrm{~cm} ; 48,21 \mathrm{~cm}$ contre 49,05 cm) (confère le tableau VII).

Tableau VII: Comparaison du poids $(\mathrm{kg})$, de la stature $(\mathrm{cm})$ et du périmètre crânien $(\mathrm{cm})$ moyens chez les enfants d'âge compris entre 37 à 49 mois de MFD à ceux de leurs pairs de

MFF

\begin{tabular}{|c|c|c|c|c|c|c|c|c|}
\hline Milieu Familial & Sexe & $\mathrm{N}$ & Poids & p-value & Stature & p-value & $\mathrm{PC}$ & $\mathrm{p}$-value \\
\hline MFD & $\bar{G}$ & 16 & $\begin{array}{r}12.85 \\
+0.34\end{array}$ & \multirow{2}{*}{$\begin{array}{c}0,762 \\
\mathrm{~ns}\end{array}$} & $\begin{array}{r}97.23 \\
+1.09\end{array}$ & \multirow{2}{*}{$0,442 \mathrm{~ns}$} & $\begin{array}{l}48.41 \\
\pm 0.38\end{array}$ & \multirow{2}{*}{$0,486 \mathrm{~ns}$} \\
\hline & $\mathrm{F}$ & 13 & $13 \pm 0,33$ & & $\begin{array}{r}96.63 \\
\pm 0,95\end{array}$ & & $48 \pm 0,42$ & \\
\hline \multirow[t]{2}{*}{ MFF } & $G$ & 21 & $\begin{array}{c}14.91 \\
\pm 0.50\end{array}$ & \multirow{2}{*}{$\begin{array}{c}0,374 \\
\text { ns }\end{array}$} & $\begin{array}{r}99.20 \\
\pm 1,35\end{array}$ & \multirow{2}{*}{$0,535 \mathrm{~ns}$} & $\begin{array}{c}49.51 \\
\pm 0,18\end{array}$ & \multirow{2}{*}{$0,542 \mathrm{~ns}$} \\
\hline & $\mathrm{F}$ & 17 & $\begin{array}{c}14.85 \\
\pm 0.44\end{array}$ & & $\begin{array}{r}99.62 \\
\pm 0.50\end{array}$ & & $\begin{array}{r}47.84 \\
\pm 0,12\end{array}$ & \\
\hline Mann-Withney & & & $0.003 \mathrm{~s}$ & & $0.118 \mathrm{~ns}$ & & $0.075 \mathrm{~ns}$ & \\
\hline
\end{tabular}

N: Effectif des sujets; G: Garçon; F: Fille; PC: Périmètre crânien; S: Différence statistiquement Significative; NS: Différence Non statistiquement Significative

Le niveau de développement physique des deux catégories enfants, à l'âge de 5 ans, est également équivalent. Ceux de milieu familial défavorisé présentent respectivement des poids, des statures et des périmètres crâniens moyens statistiquement semblables à ceux de leurs homologues favorisés $(14,55 \mathrm{~kg}$ contre $15,15 \mathrm{~kg} ; 100,13 \mathrm{~cm}$ contre $101,93 \mathrm{~cm} ; 48,84 \mathrm{~cm}$ contre 49,04 $\mathrm{cm})$ (confère le tableau VIII). 
Tableau VIII: Comparaison du poids $(\mathrm{kg})$, de la stature $(\mathrm{cm})$ et du périmètre crânien $(\mathrm{cm})$ moyens chez les enfants d'âge compris entre 50 à 59 mois de MFD à ceux de leurs pairs de MFF

\begin{tabular}{|c|c|c|c|c|c|c|c|c|}
\hline Milieu Familial & Sexe & $\mathrm{N}$ & Poids & p-value & Stature & p-value & $\mathrm{PC}$ & p-value \\
\hline MFD & $\bar{G}$ & 16 & $\begin{array}{c}14.63 \\
\pm 0.67\end{array}$ & \multirow{2}{*}{$0,063 \mathrm{~ns}$} & $\begin{array}{c}100 \\
+2.26\end{array}$ & \multirow{2}{*}{$0,500 \mathrm{~ns}$} & $\begin{array}{l}48.10 \\
\pm 0.04\end{array}$ & \multirow{2}{*}{$0,182 \mathrm{~ns}$} \\
\hline & $\mathrm{F}$ & 8 & $\begin{array}{r}14.48 \\
\pm 2,15\end{array}$ & & $\begin{array}{l}100.25 \\
\pm 2,24\end{array}$ & & $\begin{array}{c}49.58 \\
\pm 0,45\end{array}$ & \\
\hline \multirow[t]{2}{*}{ MFF } & $G$ & 5 & $\begin{array}{c}14.83 \\
\pm 0.28\end{array}$ & \multirow{2}{*}{$0,375 \mathrm{~ns}$} & $\begin{array}{l}102.12 \\
\pm 1.29\end{array}$ & \multirow{2}{*}{$0,930 \mathrm{~ns}$} & $\begin{array}{l}49.28 \\
\pm 0.74\end{array}$ & \multirow{2}{*}{$1,000 \mathrm{~ns}$} \\
\hline & $\mathrm{F}$ & 10 & $\begin{array}{r}15.48 \\
\pm 1,14\end{array}$ & & $\begin{array}{l}101.74 \\
\pm 0,71\end{array}$ & & $\begin{array}{c}48.79 \\
\pm 0,06\end{array}$ & \\
\hline Mann-Withney & & & $0.349 \mathrm{~ns}$ & & $0.572 \mathrm{~ns}$ & & $0.377 \mathrm{~ns}$ & \\
\hline
\end{tabular}

N: Effectif des sujets; G: Garçon; F: Fille; PC: Périmètre crânien; S: Différence statistiquement Significative; NS: Différence Non statistiquement Significative

L'effet non statistiquement significatif des milieux familiaux (défavorisé et favorisé) abidjanais sur le développement physique des enfants semble être constant, puisqu'il se répète en passant de l'âge de nourrisson à celui de post-nourrisson. En somme, les différents milieux familiaux (défavorisés, favorisés) offerts aux enfants de 6 à 59 mois à Koumassi et à Marcory, deux communes d'Abidjan, ne créent pas toujours, dans leur fonctionnement, des divergences pouvant influencer différemment le développement physique.

\section{Discussion}

Les observations faites auprès des enfants issus des milieux défavorisés de la commune de Koumassi et de leurs homologues des milieux favorisés aboutissent à des résultats similaires. Les enfants vivant dans un cadre de vie dépourvu d'équipements de stockage d'eau et d'évacuations d'eaux usées de qualité présentent un développement physique comparable à celui de leurs pairs qui en bénéficient.

En d'autres termes, les nourrissons et les enfants du milieu familial défavorisé de Koumassi présentent une stature, un poids et un périmètre crânien moyens qui ne diffèrent pas statistiquement de ceux de leurs homologues du milieu familial favorisé de Marcory (tableaux I, II, III et IV). La similarité du développement physique observé entre les nourrissons défavorisés et favorisés est relativement constante. Le poids, la stature et le périmètre céphalique de ces deux groupes de sujets ne varient pas un, deux ou trois ans plus tard (tableaux V, VI, VII et VIII). Il ne semble donc pas exister une différence statistiquement significative entre les deux groupes de sujets au plan du développement physique. Cette trajectoire corporelle des sujets invariable selon le milieu de vie est susceptible trouver sa source dans les stimulations socio-environnementales. 
L'un des éléments pouvant expliquer l'égalité entre le développement physique des nourrissons et des enfants de situations socio-sanitaires défavorisés et celui de leurs pairs favorisés, serait une alimentation analogue chez les deux groupes. Il faudrait indiquer que les mères des nourrissons défavorisés exercent, pour la plupart, soit des activités de commerce, de restauration, soit des activités de nettoyage des locaux des entreprises ou de balayage des rues.

Ainsi, elles quittent tôt leur domicile, abandonnant leur nourrisson ou post-nourrisson à la responsabilité de leurs petites adolescentes, nièces non scolarisées. Une proportion importante de ces enfants $(69,55 \%)$ est soumise à une alimentation structurée essentiellement de bouillie de riz, de maïs à bon marché. L'on impose à 35,45\% autres enfants un régime alimentaire composé de purée d'ignames, de bananes plantain ou de manioc. Les post-nourrissons (74\%) de conditions socio-économiques similaires ingèrent des aliments identiques, notamment les céréales, les féculents qui sont moins denses en éléments nutritifs. Les nourrissons et les post-nourrissons auraient une alimentation comparable (confère le tableau IX).

A l'image des mères défavorisées qui n'offrent leur lait que durant les trois premiers mois de vie de leur progéniture $(84,50 \%)$, celles favorisées, du fait de leurs occupations professionnelles quotidiennes, sèvrent précocement leurs enfants. Quatre-vingt-onze $(86,10 \%)$ ne bénéficient que de trois mois de lait maternel (confère le tableau IX).

Tableau IX: Comparaison de la fréquence d'allaitement et des aliments consommés par les enfants selon le milieu familial

\begin{tabular}{|c|c|c|c|c|c|c|c|}
\hline & & \multicolumn{4}{|c|}{ Allaitement pratiqué durant les 3 mois } & \multicolumn{2}{c|}{$\begin{array}{c}\text { Aliments actuels } \\
\text { consommés après 3 mois }\end{array}$} \\
\hline & & N & maternel (\%) & artificiel (\%) & mixte $(\%)$ & céréales & féculent \\
\hline \multirow{2}{*}{ MFD } & F & 66 & 85,70 & 0,00 & 14,30 & 63.20 & 36.70 \\
\cline { 2 - 8 } & G & 70 & 83,30 & 3,70 & 13,00 & 75.90 & 34.20 \\
\hline \multirow{2}{*}{ MFF } & F & 72 & 84.40 & 6.70 & 8.90 & 82.20 & 17.80 \\
\cline { 2 - 8 } & G & 70 & 87.80 & 2.40 & 9.80 & 78.00 & 21,90 \\
\hline \multirow{2}{*}{ p-value } & & & khi2=0.12 & fisher=0.001 & khi2= & khi2= & khi2=0.000 \\
& & & NS & S & $0.001 \mathrm{~S}$ & $0.000 \mathrm{~S}$ & $\mathrm{~S}$ \\
\hline
\end{tabular}

N: Effectif des sujets; G: Garçon; F: Fille; S: Différence statistiquement Significative; NS: Différence Non statistiquement Significative

Outre le non-respect du temps de sevrage, les parents favorisés sont confrontés à une multiplication irréversible des dépenses familiales (frais de scolarité, de transport, d'assurance) et à un coût élevé des compléments alimentaires infantiles. Plusieurs de leurs enfants $(80,00 \%)$ reçoivent une alimentation qui ne s'éloigne pas clairement de celle des enfants défavorisés, c'est-à-dire de bouillies de céréales, de purées de pommes de terre dont les doses en macro et en micronutriments ne correspondent pas toujours aux quantités que nécessite leur âge (confère le tableau IX). En d'autres termes, 
les nourrissons et les post-nourrissons, qu'ils soient défavorisés ou favorisés, se caractériseraient par une fréquence d'allaitement maternel équivalente et une alimentation moins fortifiée en éléments de croissance et de protection (vitamines, sels minéraux).

L'irrégularité du lait maternel et l'absence des substances nutritives dans les aliments consommés par les nourrissons et post-nourrissons favorisés pourrait entraîner chez plusieurs de ces enfants une anémie, c'est-à-dire un déficit de globules rouges, comparable à celui de leurs homologues défavorisés. Or, cette carence bio-intrinsèque ne permet pas à l'enfant de réaliser de meilleures acquisitions corporelles (Hoddinott, 2008; Ramsay, 2013). La non supériorité du développement physique des favorisés, par rapport à celui des défavorisés, serait, entre autres, due à une alimentation qui paraît identique pour les deux groupes d'enfants, contrairement à la différence d'alimentation prédite.

Les résultats obtenus dans le présent travail, loin de confirmer les prédictions faites, se révèlent spécifiques par rapport à la situation relative au développement physique des nourrissons et des enfants vivant à Abidjan (Côte d'Ivoire). Ces résultats pourraient d'autant être un aperçu du caractère unique de l'environnement physique, socio-culturel que, selon Hermanussen et al. (2001), le profil corporel des enfants est imputable aux conditions de vie offertes à ces derniers. Toutefois, la taille moins importante de l'échantillon amène à une prudence dans les conclusions. Des recherches ultérieures auprès d'effectifs de nourrissons et de post-nourrissons considérables permettraient de s'assurer de la constance ou non des présents résultats.

\section{Conclusion}

Le développement physique de l'enfant serait sous-tendu par des facteurs socio-environnementaux spécifiques. L'examen de ce lien auprès d'enfants âgés de 6 à 59 mois n'a pas permis de mettre en évidence l'effet différentiel du milieu de vie de l'enfant sur son développement physique en fonction de sa structuration. Les enfants issus de milieu familial défavorisé présentent un développement physique relativement équivalent à celui de leurs pairs vivant dans un milieu familial favorisé.

La différence de nature du milieu de vie des enfants n'entraînerait pas toujours chez ces derniers un développement physique non analogue. Les stimulations proposées par chaque milieu de vie pourraient être celles qui créent des écarts entre les enfants au plan du développement physique. En d'autres termes, la catégorie socio-professionnelle élevée des parents, les ressources financières et alimentaires dont ils disposent ne suffiraient pas pour créer pour leur progéniture de moins de 5 ans des conditions nécessaires à un développement physique supérieur à celui de leurs pairs dont les parents sont de classe basse. 


\section{Remerciements}

L'auteur du présent travail est reconnaissant à l'égard des responsables des communes de Koumassi et de Marcory pour avoir accepté que l'enquête se déroule au sein de leur commune respective. L'auteur adresse aussi ses remerciements aux différentes familles de ces communes et à leurs enfants (sujets), qui ont permis la réalisation de l'observation factuelle.

\section{References:}

1. Amor, J. C., Horton, J. R., Zhu, X., Wang, Y., Sullards, C., Ringe, D..., Kahn, R. A. (2001). Structures of yeast ARF2 and ARL1: distinct roles for the $\mathrm{N}$ terminus in the structure and function of ARF family GTPases. The journal of Biological Chemistry, 276 (45), 42477-42484.

2. Ayissi, A., Maia, C., Ayissi, J. (2002). Droits et misères de l'enfant en Afrique. Enquête au cœur d'une « invisible » tragédie. Études, 397 (10), 297-309.

3. Froment, A., Koppert, G. (2000). Malnutrition chronique et gradient climatique en milieu tropical. Paris: Editions de Bergier.

4. Hardoy, J., Mitlin, D., Sattrethwaite, D. (2001). Environmental problems in an urbanizing world finding solutions for cities in Africa, Asia and Latin America. London: Earthsan Pub.

5. Hermanussen, M., Bogin, B. (2014). Auxology-an editorial. Italian Journal of Pediatrics, 40: 8. En ligne http://www.ijponline.net/content/40/1/8.

6. Hermanussen, M., Danker-Hopfe, H., Weber, G. W. (2001). Body weight and the shape of the natural distribution of weight, in very large samples of German, Austrian and Norwegian conscripts. International Journal of Obesity, 25, 1550-1553.

7. Hioui, E. M., Farsi, Y., Aboussaleh, Y., Ahami, A. O. T. (2009). Prévalence du déficit staturo-pondéral chez les enfants préscolaires à Kenitra (Maroc). Antropo, 19, 41-55.

8. Hoddinott, J. (2008). Long-term economic effect of early childhood nutrition, The Lancet, 371 (9610), 365-366.

9. INS (1998). Recensement général de la population et de l'Habitation en 1998. Abidjan: INS.

10. INS (2012). Enquête démographique et de santé et à indicateurs multiples 2011-2012. Abidjan: INS.

11. INS (2014). Recensement Général de la Population et de l'Habitat 2014. Abidjan: INS.

12. Keino, S. G., Plasqui, G., Borne, B. V. D. (2014). Household food insecurity access: a predictor of overweight and underweight among Kenyan women. Agriculture \& Food Security, 3, 1-8. 
13. Landry, S. H. (2014). Le rôle des parents dans l'apprentissage des jeunes enfants. Texas: Children's Learning Institute, Health Science Center.

14. Müller, L., Erkman, S., Zurbrügg, C., Kouassi, D. (2010). Marcory gaz: potentiel de valorisation des déchets organiques à Abidjan. Abidjan: Centre Suisse de Recherche Scientifique.

15. Ramsay, M. (2013). Capacité à s'alimenter, appétit et comportements alimentaires des nourrissons et des jeunes enfants, et effets sur leur croissance ainsi que sur leur développement psychosocial. Montréal: McGill University.

16. Sana, C., Asma, C., Abdhakim, M., Nizar, S. (2012). Évaluation de la croissance staturo-pondérale des jeunes garçons tunisiens des régions nord et sud. Antropo, 26, 59-68.

17. Schwartz, C. E. D. (1963). Méthodes statistiques à l'usage des médecins et biologistes. Paris: Flammarion Médecine Sciences.

18. Shinsugi, C., Matsumura, M., Karama, M., Tanaka, J., Changoma, M., Kaneko, S. (2015). Factors associated with stunting among children according to the level of food insecurity in the household: a crosssectional study in a rural community of Southeastern Kenya. BioMed Central Public Health, 15, 441. doi: 10.1186/s12889-015-1802-1806.

19. Talnan, E., Fassassi, R.., Vimard, P. (2008). Pauvreté et fécondité en Côte-d'Ivoire. Pourquoi le malthusianisme de la pauvreté ne se vérifiet-il pas? Cahiers québécois de démographie, 372, 291-321.

20. Tano, J. (2000). Famille et développement cognitif. Communication au

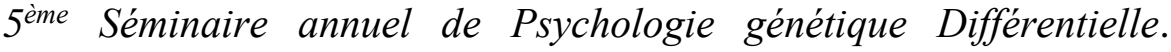
Abidjan: Université de Cocody.

21. World Health Organization (WHO, 2006). WHO child growth standards: length/height-for-age, weight-for-age, weight-for-length, weight-for-height and body mass index-for-age: methods and development. Geneva: WHO. 